\title{
Microbiological and clinical effects of adjunctive systemic metronidazole and amoxicillin in the non-surgical treatment of peri-implantitis: 1 year follow-up
}

Jamil Awad SHIBLI(a)

Daniel Sanchez FERRARI(a)

Rafael Shinoske SIROMA(a) iD

Luciene Cristina de

FIGUEIREDO ${ }^{\text {(a) }}$ iD

Marcelo de FAVERI (a) iD

Magda FERES(a)

(a) Universidade de Guarulhos - UNG, Dental Research Division, Department of Periodontology and Oral Implantology, São Paulo, SP, Brazil.

Declaration of Interests: The authors certify that they have no commercial or associative interests that represent a conflict of interest in connection with the manuscript.

Corresponding Author:

Jamil Awad Shibli

E-mail: jshibli@ung.br; jashibli@yahoo.com

htps://doi.org/10.1590/1807-3107bor-2019.vol33.0080

Submitted: June 15, 2019

Accepted for publication: July 2, 2019

Last revision: July 9, 2019

\begin{abstract}
The aim of this study was to evaluate the effects of adjunct systemic antibiotic treatment with metronidazole (MTZ) and amoxicillin (AMX) in patients receiving non-surgical subgingival debridement (NSD) for peri-implantitis. Forty subjects presenting with at least one implant with severe peri-implantitis were randomized into an experimental group [treated with NSD plus MTZ (400 mg) and AMX (500 mg) three times a day for 14 days] and a control group treated with NSD plus placebo. Clinical parameters and submucosal biofilm profiles were evaluated up to 1 year post-treatment. Overall, both treatments improved clinical parameters over time. At 1 year, mean probing depth (PD), mean clinical attachment (CA) level and proportions of red complex pathogens did not differ significantly between the two groups. In addition, mean PD and CA changes to 1-year posttreatment did not differ significantly between the two groups between baseline and 1-year post-treatment. These results suggest that the addition of MTZ and AMX to the treatment protocol of patients undergoing NSD for with severe peri-implantitis does not improve the clinical and microbiological outcomes of NSD. The fact that half of the implants in both groups did not achieve clinical success ( $\mathrm{PD}<5 \mathrm{~mm}$, no BoP, no bone loss) suggest that neither of the tested protocols were effective for treating severe peri-implantitis.
\end{abstract}

Keywords: Peri-implantitis; Amoxicillin; Metronidazole; Therapeutics.

\section{Introduction}

Systemic antibiotic therapy consisting of metronidazole (MTZ) and amoxicillin (AMX) is an effective non-surgical treatment for periodontitis. Indeed, the treatment protocol consisting of MTZ+AMX and scaling and root planing (SRP) has been shown provide additional benefits to conventional non-surgical debridement for patients with severe periodontitis, including reduced levels of key periodontal pathogens, such as Porphyromonas gingivalis, Aggregatibacter actinomycetemcomitans, and Tannerella forsythia. ${ }^{1}$ Although the microorganisms associated with the etiopathogenesis of peri-implantitis are similar to those associated with periodontitis ${ }^{2}$, to the best of our knowledge, the effectiveness of 
- Microbiological and clinical effects of adjunctive systemic metronidazole and amoxicillin in the non-surgical treatment of peri-implantitis: 1 year follow-up

MTZ+AMX treatment adjunctive to non-surgical peri-implant debridement (NSD) has not been tested before. Thus, the aim of this double-blind placebo study was to evaluate the efficacy of a treatment protocol consisting of NSD with adjunct MTZ+AMX for the treatment of peri-implantitis, relative to that of NSD alone.

\section{Methodology}

\section{Study protocol and patients}

The study protocol was developed in accordance with SPIRIT guidelines ${ }^{3}$ and approved by Guarulhos University Ethics Committee on Human Research (CEP-UNG 62/2004). Forty subjects with peri-implantitis were enrolled according to the following inclusion criteria: $\geq 35$ years of age; at least one machinedsurface implant with an external hexagon that has been functional for at least 5 years and presented peri-implantitis (probing depth $(\mathrm{PD})>5 \mathrm{~mm}$, periimplant bone loss $>4 \mathrm{~mm}$, and bleeding on probing (BoP) and/or suppuration); $\geq 50 \%$ peri-implant bone retained relative to implant length. Exclusion criteria were as follows: pregnancy, current smoking habit, any systemic disease that could affect peri-implantitis progression; NSD, antibiotic or anti-inflammatory therapy in the previous 6 months; and allergy to MTZ and/or AMX.

\section{Treatment and follow-up}

After anamneses, clinical/microbiological/ radiographic assessments, and prophylaxis/oral hygiene instruction, the participants were randomized into experimental and control groups using a computer program (https://www.randomizer.org). The experimental group (NSD-AB) patients were treated with NSD and adjunct systemic antibiotic [400 mg MTZ and $500 \mathrm{mg}$ AMX, three times a day for 14 days. The control group (NSD-P) patients underwent NSD and were given placebo MTZ and AMX pills. Antibiotics and placebos were prepared by the same pharmacy and started immediately after the first NSD session.

All NSD procedures were performed with Teflon curettes in a single appointment at baseline. All participants were treated by the same clinician
(DSF), examined by the same calibrated researcher (RSS), and given periodontal supragingival maintenance therapy every 3 months post-treatment. Microbiological assessments were performed at baseline (immediately before NSD) as well as at 14 days, 3 months, 6 months, and 1 year after NSD.

\section{Clinical and radiographic evaluation}

Each patient was examined for the presence of visible plaque, gingival bleeding, BoP and suppuration. In addition, PD (distance in $\mathrm{mm}$ from the peri-implant margin to the bottom of the pocket) and clinical attachment (distance in $\mathrm{mm}$ from the implant abutment junction to the bottom of the pocket) were checked at six sites per implant. Intra-oral x-rays were taken on Ektaspeed films according to the parallelism technique. Abutment to peri-implant bone distances were measured in scanned radiographs with Image Tool 3.0. software. These measurements were taken by a trained and calibrated examiner (JAS). A case was considered to have achieved treatment success ${ }^{4}$ if the PD was reduced to $<5 \mathrm{~mm}$ with no $\mathrm{BOP}$, and no bone loss was detected between baseline and 1 year.

\section{Microbiological evaluation}

After the clinical assessment, a submucosal sample was taken from the deepest accessible site of one diseased implant in each patient with a mini-five Gracey curette. The proportions of 39 bacterial species in each sample were determined by checkerboard DNA-DNA hybridization. The species were grouped according to the microbial complexes described by Socransky et al. ${ }^{5}$

\section{Statistical analysis}

Each clinical parameter was computed for each patient and the individual patients' values were averaged for each group. The percentage of DNA probe counts for each microbial species was determined at each site, averaged for each subject, and then averaged for each time point by group. Within-group differences between study time points were detected with the Wilcoxon Test. Between-group differences at each time point were detected with the Mann-Whitney test. The level of significance was set at 5\%. 


\section{Results}

A total of 40 patients (29 females) with mean age of $58.5 \pm 11.1$ years were enrolled in this study. The mean implant loading time (20 implants/group) was $60.5 \pm 6.3$ months. None of the clinical and microbiological parameters evaluated differed significanlty between groups at baseline (Table).

Both the NSD-AB and NSD-P treatment protocols improved all clinical parameters $(\mathrm{p}<0.05$ between baseline and 1 year) and no statistically significant differences were observed between the two groups at 1 year ( $p>0.05$, Table), including the percentage of implants achieving treatment success at 1 year $(\mathrm{PD}<5 \mathrm{~mm}$, no BoP, and no bone loss), which was $50 \%$ in the NSD-P and $65 \%$ in the NSD-AB group. Between baseline and 1 year, PD reduction was $1.8 \pm 0.2 \mathrm{~mm}$ in the NSD-P group and $3.1 \pm 1.2 \mathrm{~mm}$ in the NSD-Ab, while CA gain was $1.4 \pm 0.8 \mathrm{~mm}$ and $2.6 \pm 1.5 \mathrm{~mm}$, respectively. These differences were not statistically significant between groups.
The mean proportions of microbial complexes at baseline, at the end of antibiotic treatment (14 days), and at the 3-month, 6-month, and 1-year post-NSD follow-up time points are shown in Figure. Both therapies led to a statistically significant reduction in the proportion of red complex species at 14 days and 3 months after NSD. However, significant regrowth of red complex pathogens was observed from 3 months to 1 year post-NSD, specially in the NSD-P group. At 1 year, red complex species were still in significantly lower proportions in the test group (15.0\%) in comparison with baseline (32.7\%), but not in the control group ( $18.6 \%$ vs $24.7 \%$, respectively).

\section{Discussion}

To the best of the authors' knowledge, this study was the first to evaluate the effects of MTZ and AMX in the treatment of patients with peri-implantitis. The data did not demonstrate main clinical or microbiological benefits from the adjunctive use of these antibiotics.

Table. Clinical parameters (mean \pm SD) of test sites (i) and six implant sites (ii) in the NSD-P control group and NSD-AB experimental group ( $n=20 /$ group) during the evaluation period.

\begin{tabular}{|c|c|c|c|c|c|c|c|c|}
\hline \multirow{2}{*}{ Clinical parameter } & \multicolumn{4}{|c|}{ NSD-P control group } & \multicolumn{4}{|c|}{ NSD-AB experimental group } \\
\hline & TO & T90 & T 180 & T 365 & TO & T90 & T 180 & T 365 \\
\hline Pl i (\%) & $60.0 \pm 51.6$ & $40.0 \pm 51.6$ & $30.0 \pm 48.8$ & $50.5 \pm 53.5$ & $40.0 \pm 51.6$ & $40.0 \pm 51.6$ & $29.0 \pm 48.8^{*}$ & $68.0 \pm 37.8$ \\
\hline $\mathrm{PI}$ ii (\%) & $61.6 \pm 43.7$ & $40.0 \pm 51.6$ & $35.0 \pm 39.6$ & $61.8 \pm 41.6$ & $56.6 \pm 43.8$ & $40.0 \pm 51.6$ & $21.4 \pm 31.4^{*}$ & $49.4 \pm 30.2$ \\
\hline Gl i (\%) & $50.0 \pm 52.7$ & $10.0 \pm 31.6$ & $10.0 \pm 31.6$ & $0^{*}$ & $50.0 \pm 52.7$ & $0^{*}$ & $0^{*}$ & $0^{*}$ \\
\hline GI ii (\%) & $10.0 \pm 31.6$ & $12.0 \pm 21.4$ & $10.5 \pm 29.8$ & $4.7 \pm 12.5$ & $10.4 \pm 41.3$ & $1.6 \pm 5.2$ & $5.5 \pm 3.3$ & $3.6 \pm 12.0$ \\
\hline PD i (mm) & $7.6 \pm 1.8$ & $6.5 \pm 1.9^{*}$ & $4.3 \pm 1.8^{*}$ & $3.8 \pm 1.6^{*}$ & $9.9 \pm 3.6$ & $6.9 \pm 2.5^{*}$ & $5.2 \pm 2.1^{*}$ & $5.1 \pm 1.8^{*}$ \\
\hline PD ii (mm) & $5.5 \pm 1.3$ & $5.1 \pm 1.1$ & $3.4 \pm 0.9^{*}$ & $3.8 \pm 1.1^{*}$ & $7.0 \pm 2.6$ & $5.6 \pm 1.8^{*}$ & $3.9 \pm 1.2^{*}$ & $3.9 \pm 0.8^{*}$ \\
\hline$\Delta \mathrm{PD}$ & - & - & - & $1.8 \pm 0.2$ & - & - & - & $3.1 \pm 1.2$ \\
\hline CAL i $(\mathrm{mm})$ & $7.8 \pm 1.9$ & $6.7 \pm 2.0$ & $4.7 \pm 2.1^{*}$ & $4.5 \pm 2.0^{*}$ & $9.9 \pm 3.6$ & $7.1 \pm 2.8^{*}$ & $5.5 \pm 2.5^{*}$ & $5.7 \pm 2.6^{*}$ \\
\hline CAL ii $(\mathrm{mm})$ & $5.9 \pm 1.3$ & $5.4 \pm 1.3$ & $3.8 \pm 1.1^{*}$ & $4.4 \pm 1.4^{*}$ & $7.2 \pm 2.6$ & $5.8 \pm 1.9^{*}$ & $4.2 \pm 1.3^{*}$ & $4.2 \pm 1.0^{*}$ \\
\hline$\triangle \mathrm{CAL}$ & - & - & - & $1.4 \pm 0.8$ & - & - & - & $2.6 \pm 1.5$ \\
\hline BoP i (\%) & $97.0 \pm 34.5$ & $90.0 \pm 31.6$ & $40.0 \pm 51.6^{*}$ & $50.0 \pm 53.5^{*}$ & $90.0 \pm 31.6$ & $90.0 \pm 31.6$ & $43.0+ \pm 3.3$ & $39.0 \pm 48.8^{*}$ \\
\hline BoP ii (\%) & $85.0 \pm 18.3$ & $74.9 \pm 30.6$ & $36.6 \pm 25.8^{*}$ & $40.3 \pm 30.3^{*}$ & $86.6 \pm 32.2$ & $74.9 \pm 36.2$ & $40.4 \pm 34.5$ & $35.6 \pm 26.2^{*}$ \\
\hline Sup i (\%) & $30.0 \pm 48.3$ & $0^{*}$ & $0^{*}$ & $0^{*}$ & $50.0 \pm 52.7$ & $0^{*}$ & $0^{*}$ & $0^{*}$ \\
\hline Sup ii (\%) & $5.0 \pm 8.0$ & $0^{*}$ & $0^{*}$ & $5.3 \pm 7.1$ & $8.8 \pm 8.0$ & $0^{*}$ & $0^{*}$ & $0^{*}$ \\
\hline$\Delta \mathrm{VBL}(\mathrm{mm})$ & - & - & - & $0.47 \pm 0.31$ & - & - & - & $0.41 \pm 0.39$ \\
\hline Treatment success (\%) & - & - & - & $11(55 \%)$ & & & & $13(65 \%)$ \\
\hline
\end{tabular}

T 0: pretreatment baseline; T: 90/180/365, 90/180/365 days post-NSD; PI: plaque index; GI: gingival index; PD: probing depth; CA: clinical attachment level; BoP: bleeding on probing; Sup, suppuration; VBL: vertical Bone loss; i: data relative to the test site - probing pocket depth at the deepest site; ii: data relative to the mean of 6 sites evaluated per implant; m: mesial; d: distal; treatment success, number and percentage of subjects who achieve clinical endpoint for treatment (PD $<5 \mathrm{~mm}$, no BoP, and no bone loss). Wilcoxon Test, ${ }^{*} p<0.05$ vs. T 0 ; Mann-Whitney $U$ Test, all $p>0.05$ experimental vs. control group. 
Microbiological and clinical effects of adjunctive systemic metronidazole and amoxicillin in the non-surgical treatment of peri-implantitis: 1 year follow-up
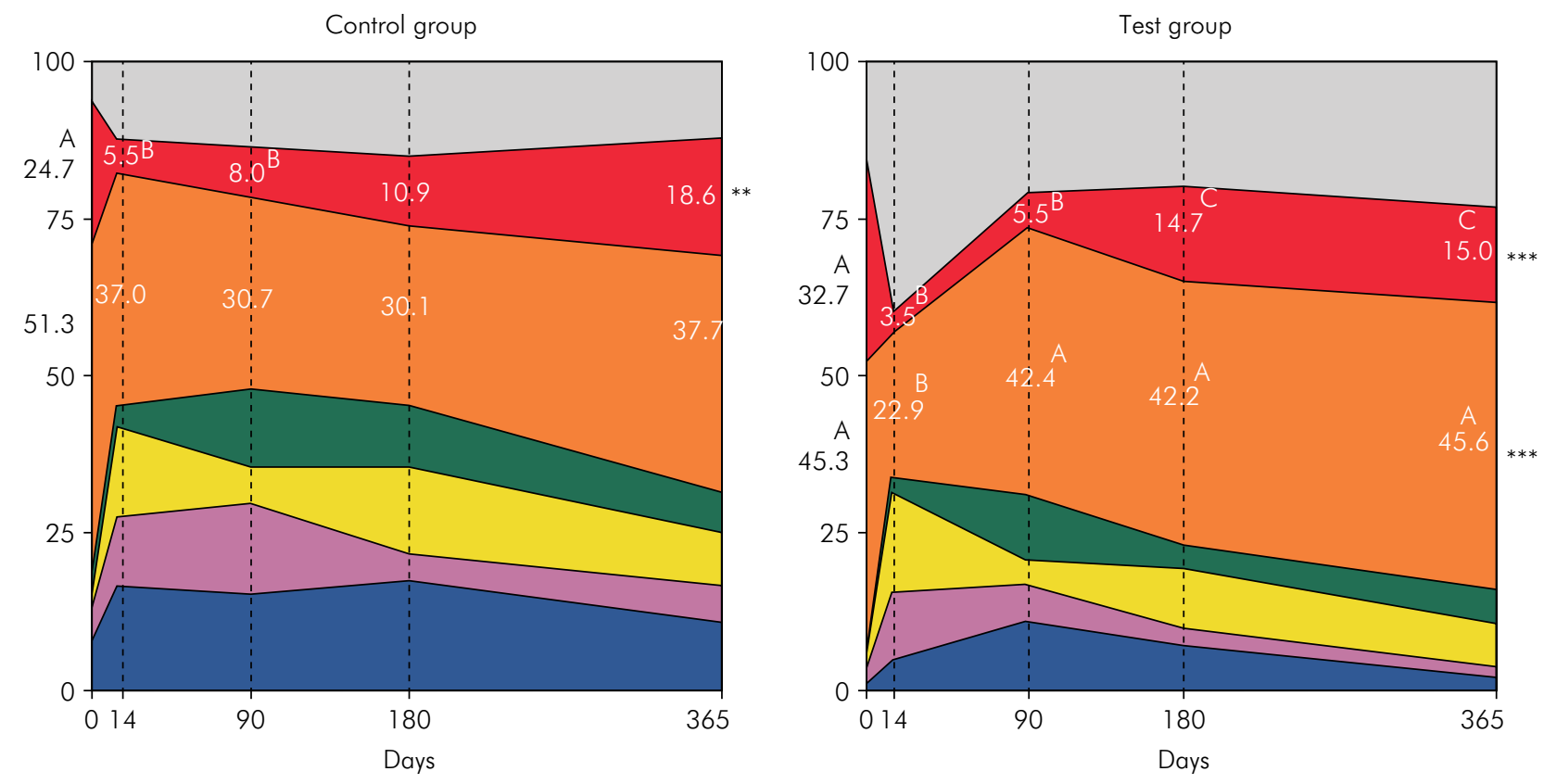

Figure. Cumulative mean proportion of microbial complexes after treatment in the subgingival plaque samples from implants. Different colors represent the microbial complexes described by Socransky et al.5 Significance of differences in cumulative mean proportions between Baseline and 12 months was sought using Wilcoxon Test (different letters represent $p<0.001$ ) and between groups at each time point using the Mann-Whitney U-test $\left({ }^{* *} p<0.05,{ }^{* * *} p<0.001\right)$.

Although not statistically significant, we did observe trends towards greater PD reduction and CA gain in the NSD-AB group than in the NSD-P group. However, overall, the percentage of implants achieving treatment success and the microbial profiles did not differ substantially between the two groups at 1 year post-treatment. The failure to achieve treatment success $(\mathrm{PD}<5 \mathrm{~mm}$, no BoP, and no bone loss) at the 1-year time point in approximetely half of the implants even in the antibiotic group indicates that non surgical debriedment may not be the most effective treatment strategy for severe peri-implantitis.

By the 1-year time-point, many patients had become recolonized with over $10 \%$ of red complex pathogens, which is considered a biological marker for clinical instability. ${ }^{1}$ These results are in agreement with previous microbiological studies that have described the difficulty of removing microbial pathogens from implant surfaces with NSD. Notably, total bacterial load has been reported previously to return to initial levels at quite short time intervals of 1-2 months and to increase gradually over time after NSD. ${ }^{6,7}$

The failure of MTZ+AMX treatment to resolve peri-implantitis despite its demonstrated efficacy in the treatment of severe periodontitis may be due to the difficulty inherent in decontaminating dental implant treads. Non-surgical peri-implant therapy has been reported to be more predictable in cases associated with moderate pockets. ${ }^{8,9}$ In this regard, it should be highlighted that the implants included in this study presented very deep pockets, many times exceeding $7 \mathrm{~mm}$. Our negative findings support the notion that severe peri-implantitis may be best treated with open-flap debridement and regenerative and/or ressective therapy. It is not known whether adjunct MTZ+AMX would improve outcomes in patients treated by open-flap debridement. Thus far, randomized clinical trials testing open-flap debridement with other antibiotic protocols, such as adjunctive AMX ${ }^{10}$ or azithromycin ${ }^{11}$, have failed to show clinically important advantages of systemic antibiotic use. To our knowledge, no previous randomized clinical trials have tested the possible benefits of prescribing MTZ and AMX to patients undergoing surgical peri-implantitis treatment, especially in severe cases. Heitz-Mayfield et al. ${ }^{12}$ demonstrated that an anti-infective protocol including surgical access, 
implant surface decontamination and systemic MTZ+AMX was effective for maintaining bone level stability or gain for up to 1 year in a study of 24 partially dentate patients with 36 implants and moderate to advanced peri-implantitis. Thus, future randomized controlled clinical trials testing the effects of MTZ+AMX adjunctive to open flap debridement may contribute to define more effective treatments for peri-implantitis.

The main limitation of this study was its small sample size. It is possible that more statistically significant results would have been obtained with a study with greater power. Nonetheless, taken together, the clinical and microbiological findings of this trial provide an important body of evidence with direct clinical applications.

In summary, the results of this study do not support the adjunctive use of systemic MTZ+AMX to the non-surgical treatment of peri-implantitis. Moreover, the achievement of clinical success ( $\mathrm{PD}<5 \mathrm{~mm}$, no BoP, and no bone loss) in only half of the patients from both treatment groups indicates that current non-surgical treatment protocols are often insufficient to treat severe peri-implantitis cases.

\section{Acknowledgement}

This study was supported by Grant FAPESP (São Paulo Research Foundation) \# 05/01939-2 and 05/03557-0

\section{References}

1. Feres M, Figueiredo LC, Soares GM, Faveri M. Systemic antibiotics in the treatment of periodontitis. Periodontol 2000. 2015 Feb;67(1):131-86. https://doi.org/10.1111/prd.12075

2. Shibli JA, Melo L, Ferrari DS, Figueiredo LC, Faveri M, Feres M. Composition of supra- and subgingival biofilm of subjects with healthy and diseased implants. Clin Oral Implants Res. 2008 Oct;19(10):975-82. https://doi.org/10.1111/j.1600-0501.2008.01566.x

3. Chan AW, Tetzlaff JM, Altman DG, Laupacis A, Gøtzsche PC, Krleža-Jerić K, et al. SPIRIT 2013 statement: defining standard protocol items for clinical trials. Ann Intern Med. 2013 Feb;158(3):200-7. https://doi.org/10.7326/0003-4819-158-3-201302050-00583

4. Heitz-Mayfield LJ, Mombelli A. The therapy of peri-implantitis: a systematic review. Int J Oral Maxillofac Implants. 2014;29 Suppl:325-45. https://doi.org/10.11607/jomi.2014suppl.g5.3

5. Socransky SS, Haffajee AD, Cugini MA, Smith C, Kent RL Jr. Microbial complexes in subgingival plaque. J Clin Periodontol. 1998 Feb;25(2):134-44. https://doi.org/10.1111/j.1600-051X.1998.tb02419.x

6. Mombelli A, Feloutzis A, Brägger U, Lang NP. Treatment of peri-implantitis by local delivery of tetracycline. Clinical, microbiological and radiological results. Clin Oral Implants Res. 2001 Aug;12(4):287-94. https://doi.org/10.1034/j.1600-0501.2001.012004287.x

7. Persson GR, Salvi GE, Heitz-Mayfield LJ, Lang NP. Antimicrobial therapy using a local drug delivery system (Arestin) in the treatment of peri-implantitis. I: microbiological outcomes. Clin Oral Implants Res. 2006 Aug;17(4):386-93. https://doi.org/10.1111/i.1600-0501.2006.01269.x

8. Faggion CM Jr, Listl S, Frühauf N, Chang HJ, Tu YK. A systematic review and Bayesian network meta-analysis of randomized clinical trials on non-surgical treatments for peri-implantitis. J Clin Periodontol. 2014 Oct;41(10):1015-25. https://doi.org/10.1111/icpe.12292

9. Salvi GE, Persson GR, Heitz-Mayfield LJ, Frei M, Lang NP. Adjunctive local antibiotic therapy in the treatment of peri-implantitis II: clinical and radiographic outcomes. Clin Oral Implants Res. 2007 Jun;18(3):281-5. https://doi.org/10.1111/j.1600-0501.2007.01377.x

10. Carcuac O, Derks J, Charalampakis G, Abrahamsson I, Wennström J, Berglundh T. Adjunctive Systemic and Local Antimicrobial Therapy in the Surgical Treatment of Peri-implantitis: A Randomized Controlled Clinical Trial. J Dent Res. 2016 Jan;95(1):50-7. https://doi.org/10.1177/0022034515601961

11. Hallström H, Persson GR, Lindgren S, Renvert S. Open flap debridement of peri-implantitis with or without adjunctive systemic antibiotics: A randomized clinical trial. J Clin Periodontol. 2017 Dec;44(12):1285-93. https://doi.org/10.1111/icpe.12805

12. Heitz-Mayfield LJ, Salvi GE, Mombelli A, Faddy M, Lang NP. Anti-infective surgical therapy of peri-implantitis. A 12-month prospective clinical study. Clin Oral Implants Res. 2012 Feb;23(2):205-10. https://doi.org/10.1111/j.1600-0501.2011.02276.x 